\title{
Asynchronous Data Recognition Research Based on Interval Association Degree
}

\author{
Jing Zhao ${ }^{\text {a) }}$, Xin Guan ${ }^{\text {b) }}$, Guidong Sun ${ }^{\text {c) }}$ and Haiqiao Liu ${ }^{\text {d) }}$ \\ Navy Aviation University, Yantai 264001, China. \\ a) Corresponding author: tt86725@163.com \\ b) 597268914@qq.com \\ c) $43458625 @ 163 . c o m$ \\ d)634256725@qq.com
}

\begin{abstract}
Aiming at the problem that the sequence data can't be fused with the interval data in the database directly, the sequence-interval asynchronous data recognition algorithm based on interval association degree was proposed. First of all, reduce the dimension of the sequence data, use the maximum and minimum of the sequence data as the interval boundary, complete the isomorphism transformation of the asynchronous data and then solve the interval similarity degree. Finally, use a recognition algorithm based on adaptive recognition criterion to deal with the in distinguishability of the recognition algorithm of the maximum association degree when association degree is fuzzy. Simulation experiments show that the proposed algorithm can recognize sequence-interval asynchronous data effectively and also discuss the recognition effect of the adaptive recognition algorithm and the maximum association degree recognition algorithm.
\end{abstract}

Key words: Target Recognition; Interval Association Degree; Sequence Data and Interval Number.

\section{INTRODUCTION}

Different sensor types produce asynchronous data types, this will make the uncertainty of information fusion increase. As a kind of uncertain data, sequence data is worth researching in the field of sensor recognition [1-3]. Due to the database recognition generally expresses as interval number, and the sequence type data detected by the sensor can't be recognized and judged directly with the data in the database. So, the research on the sensor data fusion of the sequence - interval asynchronous data can effectively solve the uncertainty of sensor recognition caused by asynchronous data and improve the recognition efficiency. The research on the recognition of the sequence - interval asynchronous data is opposite less in the field of sensor recognition. This paper starts with the measurement of the uncertainty between the two types of data, first of all, reduce the dimension of the sequence data, use the maximum and minimum of the sequence data as the interval boundary, and complete the data isomorphism transformation from the sequence type to the interval type. Then from the point of the grey association recognition theory, use interval association degree to measure the isomorphism data and get a set of interval association degree. A recognition algorithm based on the maximum association degree ordering is given [4-6]. We can get the better recognition results when the association degree level is clear, but when the association degree is similar, the recognition error will increase, so a recognition algorithm based on adaptive recognition criterion is proposed in this paper which can deal with the interval association. This algorithm can identify the interval association degree better, receive recognition results better and solve the fuzzy nature of the judgment of the maximum association degree ordering. 


\section{BASIC CONCEPTION}

\section{Standardization of the Sequence - Interval Data}

Certain sequence

$$
\boldsymbol{S}=\left(S_{1}, S_{2}, \cdots, S_{i}, \cdots, S_{n}\right)
$$

Where $S_{i}$ is the number $i$ component of the sequence, $n$ is the length of the sequence, if

$$
\begin{aligned}
& S_{-}=\min \left\{S_{i}, i=1,2, \cdots, n\right\} \\
& S_{+}=\max \left\{S_{i}, i=1,2, \cdots, n\right\}
\end{aligned}
$$

Then the sequence data can be expressed as an interval number

$$
S=\left[S_{-}, S_{+}\right]
$$

\section{Measure of Interval Number}

\section{Distance Measure of Interval Number}

If $A=\left[a_{-}, a_{+}\right]$, are two interval numbers and $a_{-} \leq a_{+}, b_{-} \leq b_{+}$, then

$$
D_{p}(A, B)=\left[\left(a_{-}-b_{-}\right)^{p}+\left(a_{+}-b_{+}\right)^{p}\right]^{\frac{1}{p}}
$$

$D_{p}(A, B)$ Is the distance between the interval number $A$ and $B$. Specially, when $p=2, D_{2}(A, B)$ is the Euclidean distance between $A$ aids $B$.

$$
D_{2}(A, B)=\left[\left(a_{-}-b_{-}\right)^{2}+\left(a_{+}-b_{+}\right)^{2}\right]^{\frac{1}{2}}
$$

\section{Association Degree of Interval Number}

The grey association coefficient between $X_{0}=\left(x_{0}(j), j=1,2, \cdots, k\right)$ and $X_{i}=\left(x_{i}(j), j=1,2, \cdots, k\right)$ is provided [7-10].

$$
r\left(x_{0}(j), x_{i}(j)\right)=\frac{\min _{i} \min _{j}\left|x_{0}(j)-x_{i}(j)\right|+\rho \cdot \max _{i} \max _{j}\left|x_{0}(j)-x_{i}(j)\right|}{\left|x_{0}(j)-x_{i}(j)\right|+\rho \cdot \max _{i} \max _{j}\left|x_{0}(j)-x_{i}(j)\right|}
$$

The association degree is

$$
\gamma\left(X_{0}, X_{i}\right)=\frac{1}{k} \cdot \sum_{j=1}^{k} r\left(x_{0}(j), x_{i}(j)\right)
$$

Then the association degree of interval number can be given according to the degree of sequence association. 


$$
\xi_{0 i}=\frac{1}{k} \cdot \sum_{j=1}^{k} \varepsilon_{0 i}(j)
$$

Where $\varepsilon_{0 i}(j)$ is the association coefficient of interval number:

$$
\varepsilon_{0 i}(j)=\frac{\min _{i} \min _{j}\left\{D_{0 i}(j)\right\}+\rho \cdot \max _{i} \max _{j}\left\{D_{0 i}(j)\right\}}{D_{0 i}(j)+\rho \cdot \max _{i} \max _{j}\left\{D_{0 i}(j)\right\}}
$$

$D_{0 i}(j)$ is the distance of interval number from $\left(x_{0-}(j), x_{0+}(j)\right)$ to $\left(x_{i-}(j), x_{i+}(j)\right), \rho$ is resolution coefficient, $\rho \in[0,1]$.

\section{RECOGNITION ALGORITHM OF THE SEQUENCE-INTERVAL ASYNCHRONOUS DATA}

\section{Algorithm Description}

Suppose that the targets to be recognized after the association processing are $m$ types. Using $T=\left\{T_{1}, T_{2}, \cdots, T_{m}\right\}$ represents the set of target sequence number. Each type of targets has $k$ types characteristic parameters which compose the feature vector set $P=\left\{P_{1}, P_{2}, \cdots, P_{k}\right\}$ of the target, it is called pattern. Assuming that the number $i(i=1,2, \cdots, m)$ target has $t_{i j}$ values in the number $j(j=1,2 \cdots, k)$ characteristic parameter. $\theta_{i j}^{n}\left(n=1,2, \cdots, t_{i j}\right)$ is the number $n$ value of the number $i$ target in the number $j$ characteristic parameter. So, each type of targets has $k$ feature vector sets. The feature vector set of the number $i$ target can be expressed as

$$
\begin{gathered}
P_{i 1}=\left(\theta_{i 1}^{1}, \theta_{i 1}^{2}, \cdots, \theta_{i 1}^{n}, \cdots, \theta_{i 1}^{t_{11}}\right) \\
P_{i 2}=\left(\theta_{i 2}^{1}, \theta_{i 2}^{2}, \cdots, \theta_{i 2}^{n}, \cdots, \theta_{i 2}^{t_{i 2}}\right) \\
\vdots \\
P_{i j}=\left(\theta_{i j}^{1}, \theta_{i j}^{2}, \cdots, \theta_{i j}^{n}, \cdots, \theta_{i j}^{t_{i 2}}\right) \\
\vdots \\
P_{i k}=\left(\theta_{i k}^{1}, \theta_{i k}^{2}, \cdots, \theta_{i k}^{n}, \cdots, \theta_{i k}^{t_{i k}}\right)
\end{gathered}
$$

Where $P_{i j}=\left\{\theta_{i j}^{n}, i=1,2, \cdots, m, j=1,2, \cdots, k, n=1,2, \cdots, t_{i j}\right\}$ expresses feature vector of the number $i$ target in the number $j$ characteristic parameter.

Then the $m$ type's characteristic parameter sets of all the targets can be expressed as

$$
\begin{gathered}
T_{p 1}=\left(P_{11}, P_{i 2}, \cdots, P_{1 j}, \cdots, P_{1 k}\right) \\
T_{p 2}=\left(P_{21}, P_{22}, \cdots, P_{2 j}, \cdots, P_{2 k}\right) \\
\vdots \\
T_{p i}=\left(P_{i 1}, P_{i 2}, \cdots, P_{i j}, \cdots, P_{i k}\right) \\
\vdots \\
T_{p m}=\left(P_{m 1}, P_{m 2}, \cdots, P_{m j}, \cdots, P_{m k}\right)
\end{gathered}
$$

Where $T_{p i}=\left\{P_{i j}, i=1,2, \cdots, m, j=1,2, \cdots, k\right\}$ expresses the characteristic parameter set of the number $i$ target. 
In general, the data in the database is the certified value plus the possible tolerance, this forms the interval database. Assuming that there are $L$ types of targets in the database and each type of targets has $k$ types of characteristic parameters. Each type of characteristic parameters is expressed as interval number, so characteristic parameter database of $L$ types of targets can be expressed as interval number

$$
\begin{gathered}
R_{1}=\left(\left[r_{11_{-}}, r_{11+}\right],\left[r_{12-}, r_{12+}\right], \cdots,\left[r_{1 j-}, r_{1 j+}\right], \cdots,\left[r_{1 k-}, r_{1 k+}\right]\right) \\
R_{2}=\left(\left[r_{21-}, r_{21+}\right],\left[r_{22-}, r_{22+}\right], \cdots,\left[r_{2 j-}, r_{2 j+}\right], \cdots,\left[r_{2 k-}, r_{2 k+}\right]\right) \\
\vdots \\
R_{l}=\left(\left[r_{l 1-}, r_{l 1+}\right],\left[r_{l 2-}, r_{l 2+}\right], \cdots,\left[r_{l j-}, r_{l j+}\right], \cdots,\left[r_{l k-}, r_{l k+}\right]\right) \\
\vdots \\
R_{L}=\left(\left[r_{L 1-}, r_{L 1+}\right],\left[r_{L 2-}, r_{L 2+}\right], \cdots,\left[r_{L j-}, r_{L j+}\right], \cdots,\left[r_{L k-}, r_{L k+}\right]\right)
\end{gathered}
$$

Where $R_{l}=\left\{\left[r_{l j-}, r_{l j+}\right], l=1,2, \cdots, L, j=1,2, \cdots, k\right\}$ expresses characteristic parameter database set of the number $l$ target, $r_{l j}=\left[r_{l j-}, r_{l j+}\right]$ expresses database interval value of the number $l$ target in the number $j$ characteristic parameter.

To solve the recognition problem of the sequence-interval asynchronous data, dealing with the measure of the asynchronous data will be the first work, which means dealing with the measure between characteristic parameter set $T_{p i}=\left\{P_{i j}, i=1,2, \cdots, m, j=1,2, \cdots, k\right\}$ and characteristic parameter database set $R_{l}=\left\{r_{l j}, l=1,2, \cdots, L, j=1,2, \cdots, k\right\}$. $P_{i j}$ performances as sequence and $r_{l j}$ performances as interval number. Asynchronous data can't measure directly, so dealing with it from the perspective of data isomorphism transformation is necessary.

\section{Algorithm Steps}

Before the isomorphism transformation, the raw data must be standardized in order to eliminate incommensurability of the sequence data of the target to be recognized which is caused by the different dimension. A variety of standardized processing methods are provided [8,9,11-13], in this paper, the means of non-dimensional generation will be used. For example, sequence $X=(x(j), j=1,2, \cdots, k)$ can be standardized as $\bar{x}(j)$, and

$$
\bar{x}(j)=\frac{x(j)}{\frac{1}{n} \cdot \sum_{j=1}^{k} x(j)}, j=1,2, \cdots, k
$$

\section{Isomorphism Transformation}

After standardization of the data, the feature vector sequence sets of the $m$ types of targets must finish isomorphism transformation between sequence and interval according to (4). Then feature vector interval sets of the $m$ types of targets will generate as follows

$$
\begin{gathered}
T p_{1}=\left(\left[p_{11_{-}}, p_{11_{+}}\right],\left[p_{12-}, p_{12+}\right], \cdots,\left[p_{1 j-}, p_{1 j_{+}}\right], \cdots,\left[p_{1 k_{-}}, p_{1 k_{+}}\right]\right) \\
T p_{2}=\left(\left[p_{21_{-}}, p_{21_{+}}\right],\left[p_{22-}, p_{22_{+}}\right], \cdots,\left[p_{2 j_{-}}, p_{2 j_{+}}\right], \cdots,\left[p_{2 k_{-}}, p_{2 k_{+}}\right]\right) \\
\vdots \\
T p_{i}=\left(\left[p_{i 1_{-}}, p_{i 1_{+}}\right],\left[p_{i 2-}, p_{i 2_{+}}\right], \cdots,\left[p_{i j-}, p_{i j+}\right], \cdots,\left[p_{i k-}, p_{i k+}\right]\right) \\
\vdots \\
T p_{m}=\left(\left[p_{m 1_{-}}, p_{m 1+}\right],\left[p_{m 2_{-}}, p_{m 2+}\right], \cdots,\left[p_{m j-}, p_{m j+}\right], \cdots,\left[p_{m k-}, p_{m k+}\right]\right)
\end{gathered}
$$


$T p_{i}=\left\{\left[p_{i j-}, p_{i j+}\right], i=1,2, \cdots, m, j=1,2, \cdots, k\right\} \quad, \quad p_{i j}=\left[p_{i j-}, p_{i j+}\right]$ expresses interval value after sequence isomorphism transformation $p_{i j-}=\min \left\{P_{i j}, i=1,2, \cdots, m, j=1,2, \cdots, k\right\}$ and $p_{i j+}=\max \left\{P_{i j}, i=1,2, \cdots, m, j=1,2, \cdots, k\right\}$ respectively expresses the upper and lower bounds of the interval sequence feature vector of the number $i$ target in the number $j$ characteristic parameter.

\section{Interval Isomorphism Association Degree Calculation}

According to (10), we can calculate the interval association coefficient $\varepsilon_{i l}(j)$ between each type of target characteristic parameter to be recognized and target characteristic parameter interval sets in the database.

It can be expressed as

$$
\varepsilon_{i l}(j)=\frac{\min _{l} \min _{j}\left\{D_{i l}(j)\right\}+\rho \cdot \max _{l} \max _{j}\left\{D_{i l}(j)\right\}}{D_{i l}(j)+\rho \cdot \max _{l} \max _{j}\left\{D_{i l}(j)\right\}}, i=1,2, \cdots, m, j=1,2, \cdots, k, l=1,2, \cdots, L
$$

Where $D_{i l}(j)$ is the interval distance between the isomorphism interval parameter to be recognized $p_{i j}=\left[p_{i j-}, p_{i j+}\right]$ and the database interval parameter $r_{l j}=\left[r_{l j-}, r_{l j+}\right]$, it can be calculated according to (6).

Through (9), interval association degree between the target characteristic parameter to be recognized and the target characteristic parameter interval set in the database can be calculated.

It can be expressed as

$$
\xi_{i l}=\frac{1}{k} \cdot \sum_{j=1}^{k} \varepsilon_{i l}(j)
$$

If the important degree of the characteristic parameter is different in the recognition processing, the weight vector of each character parameter can be determined through Delphi survey method, $\mathrm{P}$, entropy method and so on.

It can be denoted by

$$
\boldsymbol{\omega}=\left(\omega_{1}, \omega_{2}, \cdots, \omega_{j}, \cdots, \omega_{k}\right), \sum_{j=1}^{k} \omega_{j}=1
$$

Then the interval association degree can be defined as

$$
\xi_{i l}=\omega_{j} \cdot \sum_{j=1}^{k} \varepsilon_{i l}(j)
$$

\section{Recognition Based on Interval Association Degree}

Through the above processing, interval association degree set of the characteristic parameter between the target to be recognized and target database can be expressed as

$$
\xi_{i}(l)=\left\{\xi_{i l}, i=1,2, \cdots, m, l=1,2, \cdots, L\right\}
$$

$\xi_{i}(l)$ Is the interval association degree between the number $i$ target to be recognized and the number $l$ target in the database? Judge the number $i$ target to be recognized respectively belong to which type of the number $l$ target.

According to the judgment rule

$$
\begin{aligned}
& \xi_{i \operatorname{Re}(\max )}=\max \left\{\xi_{i l}, l=1,2, \cdots, L\right\} \\
& \xi_{i \operatorname{Re} c(\sec \text { ond })}=\max \left\{\xi_{i l}, l=1,2, \cdots, L, \text { and } l \neq \operatorname{Re} c(\max )\right\}
\end{aligned}
$$


And

$$
\begin{aligned}
& \xi_{i \operatorname{Re}(\max )}-\xi_{i \operatorname{Re}(\sec \text { ond })}>\sigma_{1} \\
& \xi_{i \operatorname{Re} c(\max )}>\sigma_{2}
\end{aligned}
$$

$\sigma_{1}$ Is threshold which is the correct recognition association degree exceeds the suspected one at least, $\sigma_{2}$ is threshold which is the correct recognition association degree should exceed at least. $\sigma_{1}$, can be defined as follows

$$
\begin{gathered}
\sigma_{1}=\max \left\{\frac{\sum_{l=1}^{a} \xi_{i l}}{a}, \xi_{i \operatorname{Re}(\sec \text { ond })}\right\} \\
\sigma_{2}=\sum_{\substack{l=1 \\
l \neq \operatorname{Rec}(\max ), \operatorname{Rec}(\sec \text { ond })}}^{a} \xi_{i l}
\end{gathered}
$$

If it satisfies above judgment rule, then the number $i$ target to be recognized is the number $\operatorname{Rec}(\max )$ type of the number $l$ target.

Recognition processing is as follows:

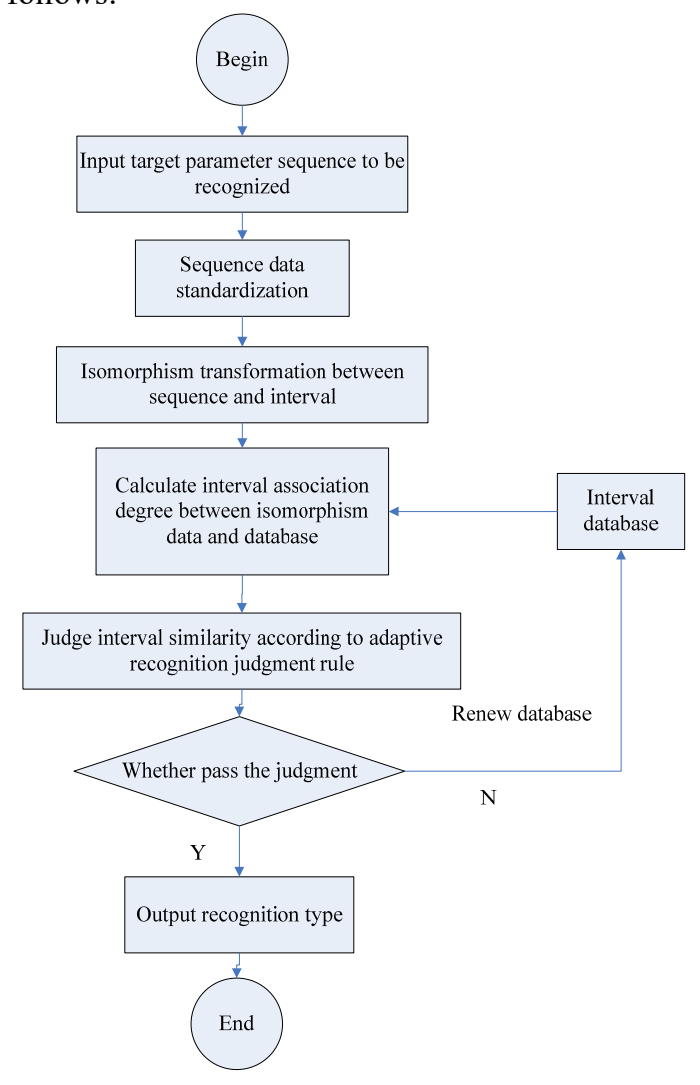

FIGURE 1. Ignition processing of sequence-interval asynchronous data. 


\section{SIMULATION ANALYSIS}

\section{Simulation Environment}

Suppose there are two types of targets to be recognized, which are expressed as $T_{1}, T_{2}$. Each type of targets has three types of characteristic parameters which are denoted by $P_{1}, P_{2}$ and $P_{3}$. Suppose that each type of targets has 100 values of each three types of characteristic parameters, so each type of targets has a vector set of characteristic parameters. There are six types of target data sets in the database, each type of targets has three types of characteristic parameters which performances interval parameter value respectively. Now we need to judge two types of targets to be recognized respectively belong to which type of the six types of targets data sets. Simulation data sequence is generated as follows

$$
\theta=\alpha+c \cdot \beta
$$

Where $\alpha$ is the discrete sequence value which obeys the uniform distribution, $\beta$ is the discrete sequence value which obeys the Gaussian distribution, $C$ is standard deviation of Gaussian distribution?

\section{Simulation Experiments}

According to the algorithm of this paper, first of all, standardize the sequence parameter of the targets to be recognized and then complete isomorphism transformation between sequence and interval. Finally, calculate interval association degree between the interval parameter of target to be recognized which has completed transformation and the interval parameter of the database. The results are shown in Fig.2.
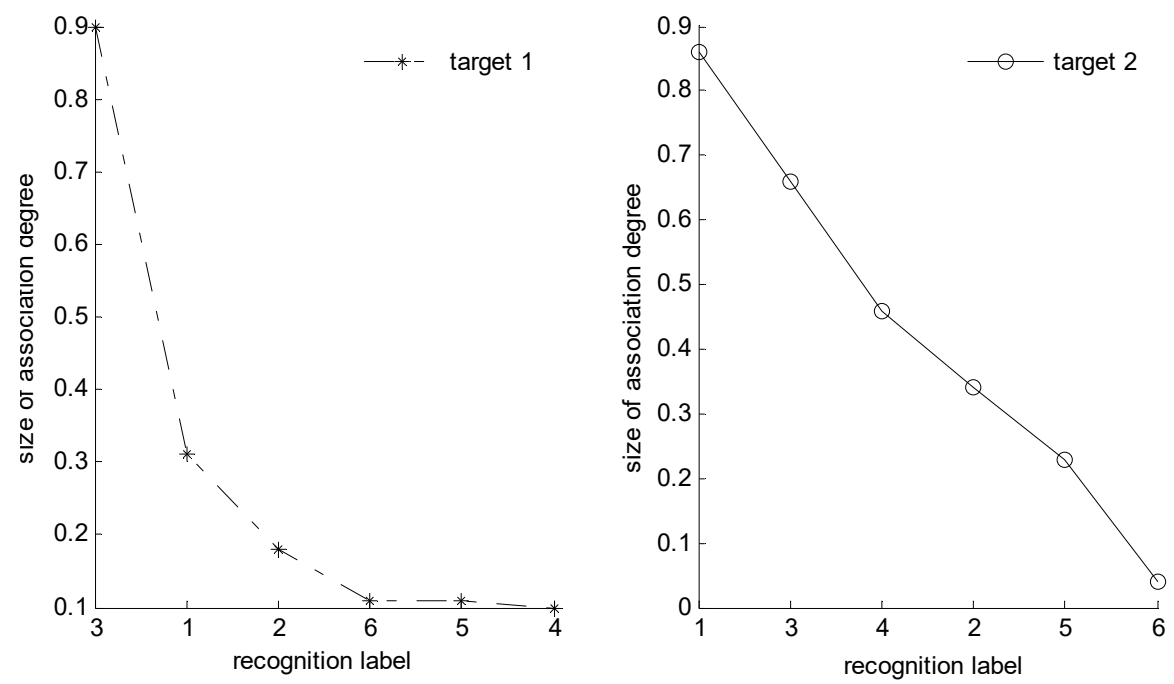

FIGURE 2. Evil association degree change chart.

In Fig.2, the coordinate represents label types in the target database, the ordinate represents interval association degree. It is shown that association degree is maximum between the first type of targets to be recognized and the third type of targets in the database. At the same time, the association degree is maximum between the second type of targets to be recognized and the first type of targets in the database.

The target can be recognized and judged by using recognition algorithm and rule based on maximum association degree, it is shown as follows

$$
\xi_{\text {max }}=\max \left(\xi_{i}, i=1,2, \cdots, l\right) \text {, and } \xi_{\text {max }}>\phi
$$


Where $\phi$ is preset parameter, $0.5 \leq \phi \leq 1$. Then the recognition results can be shown in Fig. 3 through adaptive judgment rule.

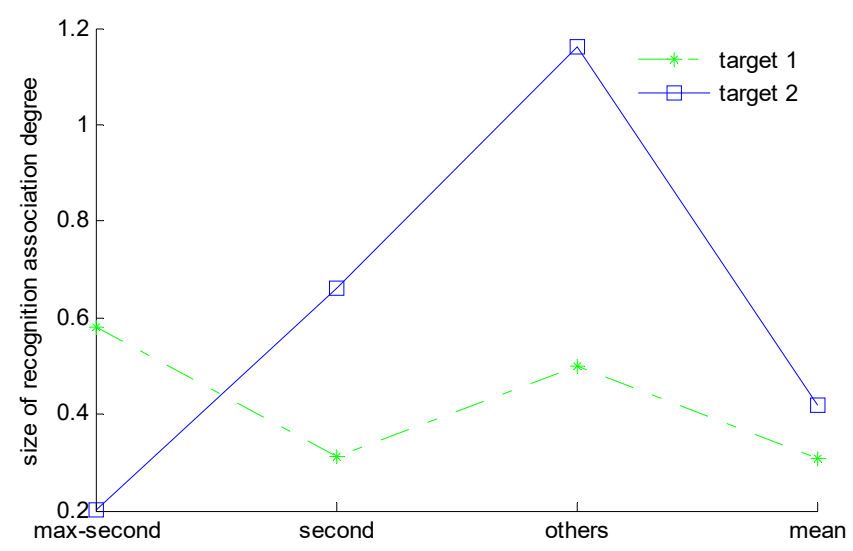

FIGURE 3. Recognition association degree change chart.

In Fig.3, the coordinate represents four recognition judgment parameters of interval association degree, they respectively express the difference between the maximum value and the second maximum value of interval association, he second maximum value of interval association, he addition of the rest association degree except the maximum value and the second maximum value, e means of interval association degree. The ordinate represents interval association degree. In Fig.3, it shows that the maximum difference exists between the first type of targets to be recognized and the first parameter in the database, so it satisfies the recognition judgment rule. But it is not the maximum difference between the second type of targets to be recognized and the first parameter in the database, so it doesn't satisfy the recognition judgment rule.

Comparison of recognition results by these two methods are shown in Table1.

TABLE 1. Comparison of recognition results.

\begin{tabular}{|c|c|c|c|c|}
\hline Recognition type & $\begin{array}{c}\text { The first type of } \\
\text { targets to be } \\
\text { recognized }\end{array}$ & $\begin{array}{c}\text { The second type of } \\
\text { targets to be } \\
\text { recognized }\end{array}$ & $\begin{array}{c}\text { The second type of } \\
\text { maximum interval } \\
\text { association degree }\end{array}$ & $\begin{array}{c}\text { The second type of } \\
\text { the second } \\
\text { maximum interval } \\
\text { association degree }\end{array}$ \\
\hline $\begin{array}{c}\text { Algorithm of this } \\
\text { paper }\end{array}$ & Target 3 & Unrecognized & \multirow{2}{*}{0.8612} & \multirow{2}{*}{0.6535} \\
\hline $\begin{array}{c}\text { Recognition } \\
\text { algorithm of } \\
\text { maximum } \\
\text { association degree }\end{array}$ & Target 3 & Target 1 & \\
\hline
\end{tabular}

In Table 1, it is shown that the first type of targets to be recognized belong to the third type of targets in the database if use the algorithm of this paper, but the second type of targets to be recognized can't find the fit recognition type in the database, so the database should be renewed and then judged. If use the recognition algorithm of maximum association degree, the first type of targets to be recognized also belong to the third type of targets in the database, but the second type of targets to be recognized belong to the first type of targets in the database. It indicates that the maximum and the second maximum of the second type of targets to be recognized are more or less similar and association degree distribution is relatively uniform. If the interval association degree is fuzzy, it will generate larger error by using the recognition algorithm of maximum association degree. So, it needs to use the algorithm of this paper to recognize and it is better to use the algorithm of this paper than the recognition algorithm of maximum association degree when the association degree is fuzzy. 


\section{CONCLUSION}

Aiming at the problem that the target sequence data parameters can't be fused directly with the interval data in the database. This paper starts with the measurement of the uncertainty between the two types data and the sequence-interval asynchronous data recognition algorithm based on interval association degree was proposed. First of all, reduce the dimension of the sequence data, use the maximum and minimum of the sequence data as the interval boundary, complete the isomorphism transformation of the asynchronous data and then solve the interval similarity degree. In this paper, use a recognition algorithm based on adaptive recognition criterion to deal with the in distinguishability of the recognition algorithm of maximum association degree when association degree is fuzzy. The algorithm of this paper can identify the interval association degree better, receive satisfactory recognition results and solve the recognition problem of the sequence-interval asynchronous data.

\section{ACKNOWLEDGMENTS}

My deepest gratitude goes first and fore most to Professor Xin Guan, my supervisor, thanks for her constant encouragement and guidance. Secondly, I also owe my sincere gratitude to my fellow classmates Guiding Sun and Haitian Liu who gave me their help and time in listening to me and helping me work out my problems during the difficult course of the thesis. Finally, thanks for the support of the National Natural Science Foundation of China (Grant No.91538201, No. 61671463 and No.61571454).

\section{REFERENCES}

1. He You, Wang Guohong and Guan Xin, Information Fusion Theory with Application (Electronic Industry Press, Beijing, 2010), pp.69.

2. Han Chongzhao, Zhu Hongyan and Duan Zhansheng, Multi-sources Information Fusion (Tsing University Press, Beijing, 2010).

3. Yang Anahi, Multi-sensor data fusion with its application (Xi'an Electronic Science and Technology University Press, Xi'an, 2004).

4. Wei Guoyu, New Method of Grey Relational Analysis to Multiple Attribute Decision Making with Intervals (Systems Engineering and Electronics, 2006), pp.1358-1359.

5. Hu Liang, Guan Xin and He You, A New Approach for Grey Multi-attribute Decision Making (Control and Decision, 2012), pp.896-898.

6. Wan Shaping, Method of Interval Deviation Degree for Uncertain Multi-sensor (Control and Decision, 2009), pp. 1306-1309.

7. Deng J L, Introduction to grey system theory (J. Grey System, 1989), pp.1 -24.

8. Deng Jalon, Elements on grey theory (Hashing Science and Technology University Press, Wu Han, 2002).

9. Liu Sighing and Dang Yoga, Grey system theory with application (Science Press, Beijing, 2010).

10. Dang Yoga and Liu Sighing, Research on Grey predicting and decision-making model (Science Press, Beijing, 2009).

11. Shingling Yue, Group decision making with multi-tribute interval data (Information Fusion, 2013), pp. 551561 .

12. Z. Xu M, Liu G and Yang N. Li, Application of interval analysis and evidence theory to fault location (IET Electric Power Applications, 2009), pp.77-84.

13. Wan Shaping, A Method for Multi-Attribute Large Group Decision Making with Interval Number Information (Pattern Recognition and Artificial, 2011), pp. 340-345. 